\title{
An inverse scattering formalism for STU supergravity
}

\author{
Despoina Katsimpouri, ${ }^{a}$ Axel Kleinschmidt ${ }^{a, b}$ and Amitabh Virmani ${ }^{c}$ \\ ${ }^{a}$ Max-Planck-Institut für Gravitationsphysik (Albert-Einstein-Institut) \\ Am Mühlenberg 1, DE-14476 Potsdam, Germany \\ ${ }^{b}$ International Solvay Institutes ULB-Campus Plaine CP231, \\ BE-1050 Brussels, Belgium \\ ${ }^{c}$ Institute of Physics \\ Sachivalaya Marg, Bhubaneshwar, Odisha, 751005 India \\ E-mail: despoina.katsimpouri@aei.mpg.de, \\ axel.kleinschmidt@aei.mpg.de, virmani@iopb.res.in
}

ABSTRACT: STU supergravity becomes an integrable system for solutions that effectively only depend on two variables. This class of solutions includes the Kerr solution and its charged generalizations that have been studied in the literature. We here present an inverse scattering method that allows to systematically construct solutions of this integrable system. The method is similar to the one of Belinski and Zakharov for pure gravity but uses a different linear system due to Breitenlohner and Maison and here requires some technical modifications. We illustrate this method by constructing a four-charge rotating solution from flat space. A generalization to other set-ups is also discussed.

KEYwords: Black Holes in String Theory, Supergravity Models, String Duality, Integrable Hierarchies

ARXIV EPRINT: 1311.7018 


\section{Contents}

1 Introduction 1

2 Preliminaries: Lagrangian and linear system 3

2.1 The three-dimensional system 3

2.2 STU gravity 3

2.3 Two-dimensional reduction and BM linear system 4

3 Riemann-Hilbert factorization for $\operatorname{SO}(4,4) \quad 6$

3.1 Solution of the Riemann-Hilbert problem $\quad 7$

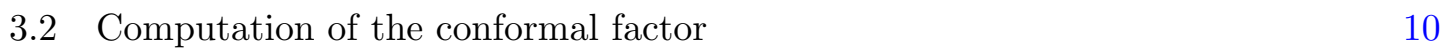

4 Construction of the four-charge black hole $\quad 11$

5 Generalization of BM method: residues of rank $r \quad 13$

6 Discussion 18

A Conventions $\quad \mathbf{1 9}$

$\begin{array}{lll}\text { A.1 The } \mathrm{SO}(4,4) \text { group and its subgroups } & 19\end{array}$

A.2 Four-dimensional metric and duality relations in $D=3 \quad 20$

$\begin{array}{ll}\text { B Two-dimensional fields for the four-charge black hole } & 21\end{array}$

\section{Introduction}

The method of inverse scattering, pioneered in gravity by Belinski and Zakharov [1-3], has been applied very successfully to pure gravity in $D=4$ and $D=5$ space-time dimensions (see also the reviews [4-6]). The method rests on identifying a linear set of equations with a spectral parameter whose compatibility yields the non-linear Einstein equation of interest. This method applies whenever one is seeking a space-time with a sufficient number of commuting and hypersurface orthogonal Killing vectors. For $D=4$ one can use inverse scattering to construct stationary and axisymmetric solutions (two Killing vectors), for $D=5$ one requires an additional space-like Killing vector to render the system integrable in the inverse scattering sense. The power of the inverse scattering method is that the construction is reduced to a purely algebraic problem for the data entering the solitonic ansatz for a solution of the linear system $[1,2]$.

There are many other gravitational systems with matter to which one would like to apply the inverse scattering method. A number of examples can be constructed from string theory where one is led to supergravity theories and the solutions sought include charged 
black holes. The class of models considered typically involves a finite-dimensional symmetry group $\mathrm{G}$ that acts as a solution generating group on the three-dimensional reduced system (one Killing vector less than for the inverse scattering method). For pure $D=4$, this group is Ehlers's $\mathrm{SL}(2, \mathbb{R})[7]$ while for maximal supergravity it is $\mathrm{E}_{8(8)}[8,9]$. A list of all such three-dimensional gravity-matter models with symmetry $\mathrm{G}$ can be found in [10]. Unfortunately, the method of inverse scattering as developed in $[1,2]$ is not directly applicable to all these cases since the soliton ansatz does not necessarily respect the structure of the group G; see for example the discussion in [11] for the case $G=G_{2(2)}$ that arises for minimal $D=5$ supergravity.

Long ago, Breitenlohner and Maison (BM) have constructed a linear system that is different from that of Belinski and Zakharov (BZ) and that takes the structure of G into account [12]. The relation between the two linear systems was studied in [11-13]. The BM linear system has not been used extensively for solution generation although in [14] it was shown how to implement a BZ like inverse scattering for $\mathrm{SL}(n, \mathbb{R})$. It is the purpose of the present article to describe how to use the BM linear system to generate solutions for more general groups $\mathrm{G}$. We will focus mainly on the case $\mathrm{G}=\mathrm{SO}(4,4)$ for concreteness. $\mathrm{G}=$ $\mathrm{SO}(4,4)$ is the symmetry that is relevant for the STU model that has multiple constructions from string theory and whose solutions have attracted a lot of attention over the years [1519]. Our methods do, however, apply more generally and we make some remarks in that direction at the end of the paper.

For the standard BZ inverse scattering method one constructs a generating function that has simple poles in the spectral parameters and the residues at these poles are of rank one. A major difference that arises for more general groups is that the rank of the residue can be larger and therefore one needs to associate more data with any given pole. We will show this explicitly for $\mathrm{G}=\mathrm{SO}(4,4)$ where the rank is two and present a general formalism in section 5. As a model example of our formalism we show how to recover the four-charge Cvetič-Youm solution [17, 20]. Rank two is sufficient in this case and we are not aware of any relevant solution of the STU model associated with higher rank. It is, however, not precluded that other interesting solutions with higher rank exist. The rank of the four-dimensional Schwarzschild solution for example in a theory with symmetry group $\mathrm{G}$ after reduction to three dimensions depends on the embedding of the standard Ehlers $\mathrm{SL}(2, \mathbb{R})$ representation into $\mathrm{G}$. For the STU model with $\mathrm{G}=\mathrm{SO}(4,4)$ this rank is two, for the theory with $\mathrm{G}=\mathrm{E}_{6}$ the rank is six and for maximal supergravity with $\mathrm{G}=\mathrm{E}_{8}$ the rank will be 57 . For other cases of interest the rank can be obtained for instance from the analysis of appendix A of [21].

The structure of this article is as follows. In section 2 we establish our conventions and review the BM linear system. In section 3, we demonstrate how to solve the linear system for $\mathrm{G}=\mathrm{SO}(4,4)$ case with rank two residues in general and work out the CvetičYoum solution as a detailed example in section 4 . Section 5 contains the general formalism for other groups and general ranks and concluding remarks can be found in section 6 . Appendix A contains some more technical details on our choice of parametrization of $\mathrm{SO}(4,4)$ in terms of the physical fields and appendix B contains the explicit expression for the scalar fields for the four-charge black hole. 


\section{Preliminaries: Lagrangian and linear system}

\subsection{The three-dimensional system}

We assume that there is a three-dimensional gravity-matter system that has a global symmetry group $\mathrm{G}$ and a local symmetry group $\mathrm{K}$ that is maximal subgroup of $\mathrm{G}$. The elements $k \in \mathrm{K}$ satisfy $k^{\#} k=1$, where the 'hash' denotes some generalized anti-involution. For $\mathrm{G}=\mathrm{SL}(n, \mathbb{R})$ and $\mathrm{K}=\mathrm{SO}(n)$ this operation is just the usual transposition $k^{\#}=k^{T}$ but it can be different in general.

The three-dimensional system is given by ${ }^{1}$

$$
\mathcal{L}_{3}=\sqrt{g_{3}}\left(R_{3}-\frac{1}{2} g^{\mu \nu} \operatorname{Tr}\left(P_{\mu} P_{\nu}\right)\right)
$$

where $P_{\mu}$ is determined by $V \in \mathrm{G} / \mathrm{K}$ through

$$
P_{\mu}=\frac{1}{2}\left(\partial_{\mu} V \cdot V^{-1}+\left(\partial_{\mu} V \cdot V^{-1}\right)^{\#}\right) .
$$

This system has the required symmetries that act on $V$ by

$$
V(x) \rightarrow k(x) V(x) g
$$

with a global $g \in \mathrm{G}$ and a local gauge transformation $k(x) \in \mathrm{K}$. A convenient object is the $x$-dependent

$$
M(x)=V^{\#}(x) V(x) \quad \text { with } \quad M(x) \rightarrow g^{\#} M(x) g
$$

and that is thus independent of the choice of gauge.

\section{$2.2 \quad$ STU gravity}

The $D=4$ STU model fits into this picture when one considers stationary solutions. In this case $\mathrm{G}=\mathrm{SO}(4,4)$ and $\mathrm{K}=\mathrm{SO}(2,2) \times \mathrm{SO}(2,2)$ [10]. The operation \# can be given a more explicit expression if one chooses to represent the scalars $V \in \mathrm{G} / \mathrm{K}$ as $(8 \times 8)$-matrices that leave invariant the metric

$$
\eta=\left(\begin{array}{ll}
0_{4} & \mathbb{1}_{4} \\
\mathbb{1}_{4} & 0_{4}
\end{array}\right),
$$

that is written in block form with unit and zero matrices. Matrices $g$ satisfying $g^{T} \eta g=$ $\eta$ belong to $\mathrm{SO}(4,4)$. The subgroup $\mathrm{K}=\mathrm{SO}(2,2) \times \mathrm{SO}(2,2)$ then satisfies the further constraint that it leaves invariant [22]

$$
\eta^{\prime}=\operatorname{diag}(-1,-1,1,1,-1,-1,1,1)
$$

and we have $V^{\#}=\eta^{\prime} V^{T} \eta^{\prime}$.

\footnotetext{
${ }^{1}$ We have changed the normalization of the scalar G/K sector by a factor of $1 / 2$ compared to [13].
} 


\subsection{Two-dimensional reduction and BM linear system}

Following the discussion in $[10,12]$, we consider further reduction of the system (2.1) over the spacelike Killing vector $\partial_{\varphi}$, thereby obtaining an effectively two-dimensional system. The three-dimensional metric can be written as

$$
d s_{3}^{2}=f^{2} d s_{2}^{2}+\rho^{2} d \varphi^{2},
$$

where the function $f$ multiplying the two-dimensional metric is called the conformal factor. Choosing Weyl coordinates $x^{m}=(\rho, z)$, the flat two-dimensional base metric is $d s_{2}^{2}=$ $d \rho^{2}+d z^{2}$. The equations of motion of the two-dimensional system read

$$
\begin{aligned}
& \pm i f^{-1} \partial_{ \pm} f=\frac{\rho}{4} \operatorname{Tr}\left(P_{ \pm} P_{ \pm}\right), \\
& D_{m}\left(\rho P^{m}\right)=0
\end{aligned}
$$

where we used the "light-cone" coordinates $x^{ \pm}=\frac{1}{2}(z \mp i \rho)$ to simplify the form of the equations. The $K$-covariant derivative is given by

$$
D_{m}=\partial_{m}-Q_{m}, \quad \text { with } \quad Q_{m}=\frac{1}{2}\left(\partial_{m} V \cdot V^{-1}-\left(\partial_{m} V \cdot V^{-1}\right)^{\#}\right) .
$$

Given a solution of $(2.8 \mathrm{~b})$, the function $f$ is obtained simply by integrating equation (2.8a). Therefore, developing a strategy to obtain solutions is mostly concentrated on equation (2.8b). In fact, this equation is shown to be integrable and can be represented by a Lax pair or linear system. This means that there exists a system of linear equations whose compatibility condition is exactly the non-linear equation we wish to solve. The functions we solve for in the linear system depend on an additional parameter $t$, called the spectral parameter.

We define the generalized coset element $\mathcal{V}(t, x)$, that has the form (suppressing the $x$-dependence)

$$
\mathcal{V}(t)=V_{0}+t V_{1}+\frac{1}{2} t^{2} V_{2}+\ldots
$$

such that

$$
\lim _{t \rightarrow 0} \mathcal{V}(t)=V_{0}:=V,
$$

and is a regular function in $t$ around $t=0$. The linear equations, referred to as the Breitenlohner-Maison (BM) linear system [12, 23]

$$
\partial_{ \pm} \mathcal{V} \mathcal{V}^{-1}=\frac{1 \mp i t}{1 \pm i t} P_{ \pm}+Q_{ \pm}
$$

can be viewed as the generalisation of the relation $\partial_{ \pm} V V^{-1}=P_{ \pm}+Q_{ \pm}$for the Lie algebravalued expression $\partial_{ \pm} V V^{-1}$, in light of the Lie algebra decomposition under the symmetric space automorphism. The integrability condition

$$
\partial_{+}\left(\partial_{-} \mathcal{V} \mathcal{V}^{-1}\right)-\partial_{-}\left(\partial_{+} \mathcal{V} \mathcal{V}^{-1}\right)-\left[\partial_{+} \mathcal{V} \mathcal{V}^{-1}, \partial_{-} \mathcal{V} \mathcal{V}^{-1}\right]=0
$$


yields the equation (2.8b) with the additional requirement that $t$ be a function which satisfies the differential equation

$$
t^{-1} \partial_{ \pm} t=\frac{1 \mp i t}{1 \pm i t} \rho^{-1} \partial_{ \pm} \rho
$$

Integrating this equation, leads to a quadratic equation for $t$ with solutions

$$
t_{ \pm}=\frac{1}{\rho}\left[(z-w) \pm \sqrt{(z-w)^{2}+\rho^{2}}\right] .
$$

The integration constant $w$ can be regarded as an alternative, $x$-independent spectral parameter. Equation (2.15) defines a two-sheeted Riemann surface over the complex $w$ plane. We choose the solution with the plus sign as the physical sheet and have $t$ to mean $t_{+}$hereafter.

The existence of the linear system (2.12) that equivalently poses the problem at hand, exhibits not only that the two-dimensional gravity system is integrable, but reveals its symmetry properties as well. The generalized coset element $\mathcal{V}(t, x)$, transforms under an enlarged symmetry group as

$$
\mathcal{V}(t) \rightarrow k(t) \mathcal{V}(t) g(w)
$$

in a manner analogous to the gauge-preserving transfomations (2.3) of $V \in \mathrm{G} / \mathrm{K}$. The general global transformation $g$ has now a dependence on the constant spectral parameter $w$ and $k(t)$ is the local compensating transformation that brings $\mathcal{V}$ back to the form (2.10). The subset of maps $g(w)$ from $S^{1} \subset \mathbb{C}$ into $\mathrm{G}$ constitute the loop group $\hat{\mathrm{G}}$. This already shows that the symmetry group of the two-dimensional system includes the infinitedimensional loop group associated to the finite group G. In fact, the group of transformations involves the full affine extension of $\mathrm{G}$, which comprises the central extension acting on the conformal factor $f[12]$.

The symmetric space automorphism \# admits a generalization for the enlarged symmetry group and its action on the functions $\mathcal{V}(t)$ is given by

$$
(\mathcal{V}(t))^{\#}=\mathcal{V}^{\#}\left(-\frac{1}{t}\right)
$$

With this definition, it can be shown that for any solution $\mathcal{V}$ of (2.12) the quantity $\partial_{ \pm} \mathcal{V} \mathcal{V}^{-1}$ is anti-invariant under the \# -involution induced on the associated Lie algebra. This means that if $\mathcal{V}(t)$ is a solution of $(2.12)$, then the function $(\mathcal{V}(t))^{\#}$ is also a (generally distinct) solution.

In principle, given a seed solution $\mathcal{V}(t)$ one could obtain new solutions $\mathcal{V}^{g}(t)$ through the transformation (2.16). However, in this approach one needs to determine $k(t)$, a task that is generally quite hard. Alternatively, we can construct a function, analogous to $M=V^{\#} V$, called the monodromy matrix

$$
\mathcal{M}(w)=(\mathcal{V}(t))^{\#} \mathcal{V}(t)=\mathcal{V}^{\#}\left(-\frac{1}{t}\right) \mathcal{V}(t)
$$


which transforms as

$$
\mathcal{M}(w) \rightarrow \mathcal{M}^{g}(w):=g^{\#}(w) \mathcal{M}(w) g(w),
$$

thus evading knowledge of the element $k(t)$. The \#-properties of (2.12) imply that $\mathcal{M}(w)$ is constant: $\partial_{ \pm} \mathcal{M}(w)=0$. Solutions can be now obtained from the factorization of $\mathcal{M}^{g}(w)$ into $\left(\mathcal{V}^{g}(t)\right)^{\#} \mathcal{V}^{g}(t)$. This is a Riemann-Hilbert problem, that is generally difficult to solve. However, in special circumstances, it becomes a purely algebraic procedure, as described in the following section. Generally, the physical fields can be obtained from $\mathcal{V}^{g}(t)$ by taking the limit $t \rightarrow 0$. On top of the solution of the Riemann-Hilbert problem (2.18) we also need to determine the conformal factor $f$ by integrating (2.8a). In the algebraic case considered in the next section this is also easy to accomplish.

As in our previous work [13], in this article we always work with flat space

$$
\mathcal{V}(t)=\mathbb{1} \quad \text { and } \quad f=1,
$$

as seed solution. Thus, from now on we simply drop the superscript $g$ from $\mathcal{M}^{g}(w)$ and $\mathcal{V}^{g}$ and think of being given a monodromy matrix $\mathcal{M}(w)$ that needs to be factorized to find $\mathcal{V}(t)$.

\section{Riemann-Hilbert factorization for $\mathrm{SO}(4,4)$}

We construct the monodromy matrix $\mathcal{M}$ as

$$
\mathcal{M}=\mathcal{V}^{\#}\left(-\frac{1}{t}, x\right) \mathcal{V}(t, x)=\eta^{\prime} \mathcal{V}^{T}\left(-\frac{1}{t}, x\right) \eta^{\prime} \mathcal{V}(t, x)
$$

where $\eta^{\prime}$ is the quadratic form of (2.6) preserved by $\mathrm{SO}(2,2) \times \mathrm{SO}(2,2)$ and

$$
g^{\#}=\eta^{\prime} g^{T} \eta^{\prime-1}, \quad \forall \quad g \in \mathrm{SO}(4,4) .
$$

The matrix $\mathcal{M}$ is by construction an element in $\mathrm{SO}(4,4)$ (as $\mathcal{V} \in \mathrm{SO}(4,4)$ ). As mentioned in the previous section, involution symmetry together with the Lax equations imply that $\partial_{\mu} \mathcal{M}=0$, i.e., $\mathcal{M}$ is independent of the spacetime coordinates $(\rho, z)$ and is a function of $w$ alone $[12,23]$. Since $w$ is invariant under $t \rightarrow-1 / t$, it follows that $\mathcal{M}$ is also invariant under simultaneous action of the generalized transposition \# and the exchange $t \rightarrow-1 / t$ :

$$
\mathcal{M}^{\#}=\eta^{\prime} \mathcal{V}^{T}(t, x) \eta^{\prime} \mathcal{V}\left(-\frac{1}{t}, x\right)=\mathcal{M}
$$

In order to find $\mathcal{V}(t)$ from $\mathcal{M}$, we wish to factorize the matrix $\mathcal{M}$ in the form

$$
\mathcal{M}(w)=A_{-}^{\#}(t, x) M(x) A_{+}(t, x)
$$

with $A_{+}(t)$ containing only positive powers of $t[12,14]$ and where the matrices $A_{ \pm}$satisfy the relation $[13,14]$

$$
A_{-}(t, x)=A_{+}\left(-\frac{1}{t}, x\right),
$$

and $M^{\#}(x)=M(x)$. We also require matrices $A_{ \pm}(t, x)$ to be in $\mathrm{SO}(4,4)$. Furthermore we factorize $M(x)=V^{\#}(x) V(x)$ so that

$$
\mathcal{V}(t, x)=V(x) A_{+}(t, x) .
$$




\subsection{Solution of the Riemann-Hilbert problem}

We restrict ourselves to the class of matrices $\mathcal{M}(w)$ that have $N$ simple poles at locations $w=w_{k}$ that can be expressed in the form,

$$
\begin{aligned}
\mathcal{M}(w) & =\mathbb{1}+\sum_{k=1}^{N} \frac{A_{k}}{w-w_{k}}, \\
\mathcal{M}^{-1}(w) & =\eta \mathcal{M}^{T} \eta=\eta\left(\mathbb{1}+\sum_{k=1}^{N} \frac{A_{k}^{T}}{w-w_{k}}\right) \eta .
\end{aligned}
$$

The matrix $\eta$ is the quadratic form preserved by $\mathrm{SO}(4,4)$.

Unlike the case of $\operatorname{SL}(n, \mathbb{R})$ considered in $[13,14]$ where the residue matrices $A_{k}$ are taken to be of rank one, in the present analysis we take the residue matrices $A_{k}$ to be of rank two. In the following, in particular in the next section, it will become clear that the rank-two case corresponds to the simple solutions of physical interest. An intuitive way to appreciate this is via the restriction of the general $\mathrm{SO}(4,4)$ matrix $M(x)$ to fourdimensional vacuum gravity. The structure of the restricted matrix is such that the Ehlers SL(2) representative of four-dimensional vacuum gravity enters two times, suggesting that the residue matrices in $\mathcal{M}(w)$ should be taken to be of rank two in order to connect to solutions of vacuum gravity. A related observation was also made in [11], where in the context of the BZ method it was pointed out that for minimal supergravity, soliton transformations must be applied in pairs in order to preserve the coset structure.

Using the expression

$$
\frac{1}{w-w_{k}}=\nu_{k}\left(\frac{t_{k}}{t-t_{k}}+\frac{1}{1+t t_{k}}\right),
$$

where $t_{k}$ is the value of (2.15) at $w=w_{k}$, and

$$
\nu_{k}=-\frac{2}{\rho\left(t_{k}+\frac{1}{t_{k}}\right)},
$$

we can write

$$
\mathcal{M}(t, x)=\mathbb{1}+\sum_{k=1}^{N} \frac{\nu_{k} t_{k} A_{k}}{t-t_{k}}+\sum_{k=1}^{N} \frac{\nu_{k} A_{k}}{1+t t_{k}} .
$$

The residue matrices $A_{k}$ can be factorized and parameterized as follows,

$$
A_{k}=\alpha_{k} a_{k} a_{k}^{T} \eta^{\prime}-\beta_{k}\left(\eta b_{k}\right)\left(\eta b_{k}\right)^{T} \eta^{\prime},
$$

where $a_{k}$ and $b_{k}$ are 8-dimensional constant vectors. At first sight this choice may not look transparent or obvious, but its advantages will become clear very soon. Note that by construction, the matrices $A_{k}(3.11)$ satisfy

$$
A_{k}^{\#}=A_{k},
$$


as they should, since $\mathcal{M}(w)$ satisfies this property. In order to deduce properties of the vectors $a_{k}$ and $b_{k}$, we study the pole structure of the product $\mathcal{M}(t, x) \mathcal{M}^{-1}(t, x)$ or equivalently the pole structure of $\mathcal{M}(t, x) \eta \mathcal{M}^{T}(t, x)$. The absence of double poles in this product at $t=-1 / t_{k}$ implies the conditions

$$
A_{k} \eta A_{k}^{T}=0 \text { for all } k \text {. }
$$

These conditions are fulfilled when the vectors satisfy the following relations,

$$
\begin{aligned}
a_{k}^{T} \eta a_{k} & =0, \\
b_{k}^{T} \eta b_{k} & =0, \\
a_{k}^{T} b_{k} & =0,
\end{aligned}
$$

for all $k$. The absence of single poles in the product $\mathcal{M}(t, x) \eta \mathcal{M}^{T}(t, x)$ at $t=-1 / t_{k}$ results in the conditions

$$
\mathcal{A}_{k} \eta A_{k}^{T}=-A_{k} \eta \mathcal{A}_{k}^{T}
$$

where matrices $\mathcal{A}_{k}$ are defined as

$$
\mathcal{A}_{k}=\left.\left(\mathcal{M}(t, x)-\frac{\nu_{k} A_{k}}{1+t t_{k}}\right)\right|_{t \rightarrow-\frac{1}{t_{k}}} .
$$

The condition (3.15) explicitly reads

$$
\mathcal{A}_{k} \eta \eta^{\prime} \alpha_{k} a_{k} a_{k}^{T}-\mathcal{A}_{k} \eta \eta^{\prime} \beta_{k}\left(\eta b_{k}\right)\left(\eta b_{k}\right)^{T}=-\alpha_{k} a_{k} a_{k}^{T} \eta^{\prime} \eta \mathcal{A}_{k}^{T}+\beta_{k}\left(\eta b_{k}\right)\left(\eta b_{k}\right)^{T} \eta^{\prime} \eta \mathcal{A}_{k}^{T} .
$$

A sufficient condition for these equations is the existence of (space-time dependent) numbers $\gamma_{k}$ such that

$$
\begin{aligned}
\mathcal{A}_{k} \eta \eta^{\prime} a_{k} & =\nu_{k} \beta_{k} \gamma_{k}\left(\eta b_{k}\right), \\
\left(\eta b_{k}\right)^{T} \eta^{\prime} \eta \mathcal{A}_{k}^{T} & =\nu_{k} \alpha_{k} \gamma_{k} a_{k}^{T} .
\end{aligned}
$$

Recall that, in order to solve the Riemann-Hilbert problem, we wish to factorize the matrix $\mathcal{M}$ in the form

$$
\mathcal{M}(w)=A_{-}^{\#}(t, x) M(x) A_{+}(t, x)
$$

with matrices $A_{ \pm}$satisfying the relation

$$
A_{-}(t, x)=A_{+}\left(-\frac{1}{t}, x\right)
$$

and $M^{\#}(x)=M(x)$. We also require matrices $A_{ \pm}(t, x)$ to be matrices in $\mathrm{SO}(4,4)$. Furthermore we factorize $M(x)=V^{\#}(x) V(x)$ so that

$$
\mathcal{V}(t, x)=V(x) A_{+}(t, x)
$$


The analyticity properties (2.10) of the resulting $\mathcal{V}(t, x)$ in the neighbourhood of $t=0$ require that the poles at $t=-1 / t_{k}$ come from the factor $A_{+}[12,14]$. We therefore make the ansätze generalizing the ones used in $[13,14]$

$$
A_{+}(t)=\mathbb{1}-\sum_{k=1}^{N} \frac{t C_{k}}{1+t t_{k}}
$$

with the parametrization of matrices $C_{k}$ as follows

$$
C_{k}=c_{k} a_{k}^{T} \eta^{\prime}-\left(\eta d_{k}\right)\left(\eta b_{k}\right)^{T} \eta^{\prime}
$$

As in the $\mathrm{SL}(n, \mathbb{R})$ case, the vectors $a_{k}, b_{k}, c_{k}$, and $d_{k}$ are not all independent and determining their relation amounts to solving the Riemann-Hilbert problem.

In order to determine the vectors $c_{k}$ and $d_{k}$ we study the poles in the product $A_{+}(t) \eta \mathcal{M}^{T}(t, x)$ at $t=-1 / t_{k}$. The condition for no double poles is

$$
C_{k} \eta A_{k}^{T}=0
$$

which is fulfilled when the conditions (3.14) hold. Furthermore, we need to ensure that the product $A_{+}(t) \eta \mathcal{M}^{T}(t, x)$ has no single poles at $t=-1 / t_{k}$. This requirement is equivalent to

$$
t_{k}^{-1} C_{k} \eta \mathcal{A}_{k}^{T}+\left.\left(A_{+}+\frac{t C_{k}}{1+t t_{k}}\right)\right|_{t=-\frac{1}{t_{k}}} \eta \nu_{k} A_{k}^{T}=0
$$

Writing equation (3.25) in terms of the vectors $a_{k}, b_{k}, c_{k}$, and $d_{k}$ and using relations (3.18a) and $(3.18 \mathrm{~b})$, we arrive at

$$
\begin{aligned}
& t_{k}^{-1}\left(c_{k} \nu_{k} \beta_{k} \gamma_{k}\left(\eta b_{k}\right)^{T}-\left(\eta d_{k}\right) \nu_{k} \alpha_{k} \gamma_{k} a_{k}^{T}\right)+\nu_{k} \alpha_{k} \eta \eta^{\prime} a_{k} a_{k}^{T}-\nu_{k} \beta_{k} \eta \eta^{\prime}\left(\eta b_{k}\right)\left(\eta b_{k}\right)^{T} \\
& \quad+\sum_{\substack{l=1 \\
l \neq k}}^{N} \frac{1}{t_{k}-t_{l}}\left(c_{l} a_{l}^{T} \eta^{\prime}-\left(\eta d_{l}\right)\left(\eta b_{l}\right)^{T} \eta^{\prime}\right) \eta \nu_{k}\left(\eta^{\prime} \alpha_{k} a_{k} a_{k}^{T}-\eta^{\prime} \beta_{k}\left(\eta b_{k}\right)\left(\eta b_{k}\right)^{T}\right)=0
\end{aligned}
$$

This condition is satisfied when the following two conditions are satisfied

$$
-t_{k}^{-1}\left(\eta d_{k}\right) \nu_{k} \alpha_{k} \gamma_{k}+\nu_{k} \alpha_{k} \eta \eta^{\prime} a_{k}+\sum_{\substack{l=1 \\ l \neq k}}^{N} \frac{\nu_{k} \alpha_{k}}{t_{k}-t_{l}}\left(c_{l} a_{l}^{T} \eta a_{k}-\left(\eta d_{l}\right)\left(\eta b_{l}\right)^{T} \eta a_{k}\right)=0,
$$

and

$$
t_{k}^{-1} c_{k} \nu_{k} \beta_{k} \gamma_{k}-\nu_{k} \beta_{k} \eta \eta^{\prime}\left(\eta b_{k}\right)-\sum_{\substack{l=1 \\ l \neq k}}^{N} \frac{\nu_{k} \beta_{k}}{t_{k}-t_{l}}\left(c_{l} a_{l}^{T} \eta\left(\eta b_{k}\right)-\left(\eta d_{l}\right)\left(\eta b_{l}\right)^{T} \eta\left(\eta b_{k}\right)\right)=0 .
$$

Assuming furthermore that the vectors $a_{k}, b_{k}$ satisfy

$$
\begin{aligned}
& a_{l}^{T} \eta a_{k}=0, \\
& b_{l}^{T} \eta b_{k}=0,
\end{aligned}
$$


for $l \neq k$, then the relations (3.27) and (3.28) simplify to

$$
\begin{aligned}
\eta^{\prime} a_{k} & =\frac{\gamma_{k}}{t_{k}} d_{k}+\sum_{l \neq k}^{N} \frac{1}{t_{k}-t_{l}} d_{l}\left(a_{k}^{T} b_{l}\right), \\
\eta^{\prime} b_{k} & =\frac{\gamma_{k}}{t_{k}} c_{k}+\sum_{l \neq k}^{N} \frac{1}{t_{l}-t_{k}} c_{l}\left(a_{l}^{T} b_{k}\right) .
\end{aligned}
$$

These relations can be written as matrix equations

$$
\begin{aligned}
\eta^{\prime} a & =d \Gamma^{T}, \\
\eta^{\prime} b & =c \Gamma,
\end{aligned}
$$

where $a, b, c$, and $d$ are $8 \times N$ matrices whose columns are the vectors $a_{k}, b_{k}, c_{k}, d_{k}$ respectively and $\Gamma$ is a $N \times N$ matrix with elements

$$
\Gamma_{k l}=\left\{\begin{array}{lll}
\frac{\gamma_{k}}{t_{k}} & \text { for } & k=l \\
\frac{a_{k}^{T} b_{l}}{t_{k}-t_{l}} & \text { for } & k \neq l .
\end{array}\right.
$$

Solving equations (3.32a) and (3.32b) for $c$ and $d$ we find the matrix $A_{+}(t, x)$ as

$$
A_{+}(t)=\mathbb{1}-\eta^{\prime} b \Gamma^{-1} \frac{t}{\mathbb{1}+t T} a^{T} \eta^{\prime}+\eta \eta^{\prime} a\left(\Gamma^{T}\right)^{-1} \frac{t}{\mathbb{1}+t T} b^{T} \eta \eta^{\prime},
$$

where to avoid notational clutter we use $T$ to denote the $N \times N$ diagonal matrix with entries $t_{k}$. Taking the limit of the inverse of (3.34) as $t \rightarrow \infty$ we get the matrix $M(x)$,

$$
M(x)=A_{+}^{-1}(\infty)=\eta A_{+}^{T}(\infty) \eta,
$$

with

$$
A_{+}^{T}(\infty)=\mathbb{1}-\eta^{\prime} a T^{-1}\left(\Gamma^{-1}\right)^{T} b^{T} \eta^{\prime}+\eta^{\prime} \eta b T^{-1} \Gamma^{-1} a^{T} \eta^{\prime} \eta .
$$

If we furthermore assume that $a_{l}^{T} b_{k}=-a_{k}^{T} b_{l}$ for $l \neq k$, i.e., that the $\Gamma$ matrix is symmetric, then expression (3.36) becomes

$$
A_{+}^{T}(\infty)=\mathbb{1}-\eta^{\prime} a T^{-1} \Gamma^{-1} b^{T} \eta^{\prime}+\eta^{\prime} \eta b T^{-1} \Gamma^{-1} a^{T} \eta^{\prime} \eta .
$$

In the next section, we see that all assumptions made in the above analysis are satisfied for the four-charge black holes - one of most studied set-up in four-dimensional STU supergravity. We believe that various assumptions made above are also satisfied in more general settings of physical interest.

\subsection{Computation of the conformal factor}

The conformal factor is determined by integration of equation (2.8a). This proceeds exactly along the same lines as in appendix A of [13], keeping in mind the change of normalization of the scalars, cf. footnote 1 . We do not repeat all the steps here but only indicate a few intermediate results where the rank-two property of the residues enters. 
For evaluating (2.8a) we need to detemine $\operatorname{Tr}\left(P_{ \pm} P_{ \pm}\right)$. This is most conveniently done in terms of evaluating first $A_{+}^{-1}(t) \frac{\partial}{\partial t} A_{+}(t)[13,14]$. For the value of $A_{+}(t)$ determined in (3.34) one finds

$$
A_{+}^{-1}(t) \frac{\partial}{\partial t} A_{+}(t)=-\eta^{\prime} b \frac{\mathbb{1}}{\mathbb{1}+t T} \Gamma^{-1} \frac{\mathbb{1}}{\mathbb{1}+t T} a^{T} \eta^{\prime}+\eta \eta^{\prime} a \frac{\mathbb{1}}{\mathbb{1}+t T} \Gamma^{-1} \frac{\mathbb{1}}{\mathbb{1}+t T} b^{T} \eta \eta^{\prime},
$$

which is now composed of two terms reflecting the rank-two nature of the residues. The next important intermediate quantity is

$$
\operatorname{Tr}\left(A_{+}^{-1}( \pm i) \dot{A}_{+}( \pm i)\right)^{2}=2 \sum_{k, l, m, n} \frac{\Gamma_{k l}^{-1} \Gamma_{m n}^{-1}}{\left(1 \pm i t_{k}\right)\left(1 \pm i t_{l}\right)\left(1 \pm i t_{m}\right)\left(1 \pm i t_{n}\right)} \operatorname{Tr}\left(b_{k} a_{l}^{T} b_{m} a_{n}^{T}\right)
$$

where the factor of 2 is due to the increased rank. Otherwise the result is exactly equal to the one in [13]. The changed normalization of the scalars cancels this factor of 2 so that we obtain the conformal factor as

$$
f^{2}=k_{\mathrm{BM}} \cdot \prod_{k=1}^{N}\left(t_{k} \nu_{k}\right) \cdot \operatorname{det} \Gamma
$$

where $k_{\mathrm{BM}}$ is an integration constant.

\section{Construction of the four-charge black hole}

In this section we present a fairly non-trivial implementation of the inverse scattering method of the previous section. We construct the four-charge black hole of STU supergravity from flat space. This construction illustrates all the steps of the algorithm presented earlier.

As in the $\mathrm{SL}(n, \mathbb{R})$ case studied in $[13,14]$ the main difficulty in constructing the general multisoliton solutions using the BM method lies in finding the appropriate meromorphic matrices $\mathcal{M}(w)$ that satisfy the various requirements of the previous section and satisfy the coset constraints. It turns out that in the two-soliton case, as in the $\operatorname{SL}(n, \mathbb{R})$ models, finding appropriate solitonic matrices is not difficult. We start with monodromy matrices of the form

$$
\mathcal{M}(w)=\mathbb{1}+\frac{A_{1}}{w-c}+\frac{A_{2}}{w+c}
$$

where

$$
\begin{aligned}
& A_{1}=\alpha_{1} a_{1} a_{1}^{T} \eta^{\prime}-\beta_{1}\left(\eta b_{1}\right)\left(\eta b_{1}\right)^{T} \eta^{\prime}, \\
& A_{2}=\alpha_{2} a_{2} a_{2}^{T} \eta^{\prime}-\beta_{2}\left(\eta b_{2}\right)\left(\eta b_{2}\right)^{T} \eta^{\prime},
\end{aligned}
$$

and where $a_{1}, a_{2}$ and $b_{1}, b_{2}$ are 8-dimensional vectors. In writing (4.1) the location of the poles is chosen to be at $w_{1}=+c$ and $w_{2}=-c$. This choice can always be made by 'shifting' the axis (see [13] for a more detailed discussion on this). For finding the vectors $a_{1}, a_{2}$ and $b_{1}, b_{2}$ corresponding to the four-charge black hole, let us start by looking at corresponding vectors for the Kerr-black hole in the $\mathrm{SO}(4,4)$ context. Analyzing the structure of the 
$\mathrm{SO}(4,4)$ matrix $M(x)$ and embedding of the Ehlers's $\mathrm{SL}(2, \mathbb{R})$ in it, we make the inspired ansatz for the $a$-vectors

$$
\begin{aligned}
& a_{1}=(0,0,-\zeta, 0,0,0,0,1)^{T}, \\
& a_{2}=(0,0,1,0,0,0,0,-\zeta)^{T} .
\end{aligned}
$$

Next we follow an algorithm similar to the one used in $[13,14]$ to construct the $b$-vectors. We first construct the matrix $a=\left(a_{1}, a_{2}\right)$, next we find the $2 \times 2$ matrix $\xi=a^{T} \eta^{\prime} a$ and choose

$$
b=(\sqrt{\operatorname{det} \xi}) \eta^{\prime} a \xi^{-1} \epsilon \quad \text { with } \quad \epsilon=\left(\begin{array}{cc}
0 & -1 \\
1 & 0
\end{array}\right) .
$$

This results in $b$-vectors

$$
\begin{aligned}
& b_{1}=(0,0,1,0,0,0,0, \zeta)^{T} \\
& b_{2}=(0,0,-\zeta, 0,0,0,0,-1)^{T}
\end{aligned}
$$

Finally we must choose

$$
\begin{array}{ll}
\alpha_{1}=+2 c \frac{1+\zeta^{2}}{\left(1-\zeta^{2}\right)^{2}}, & \alpha_{2}=-2 c \frac{1+\zeta^{2}}{\left(1-\zeta^{2}\right)^{2}}, \\
\beta_{1}=-2 c \frac{1+\zeta^{2}}{\left(1-\zeta^{2}\right)^{2}}, & \beta_{2}=+2 c \frac{1+\zeta^{2}}{\left(1-\zeta^{2}\right)^{2}},
\end{array}
$$

in order to satisfy the coset constraints. It can be readily verified that all the conditions required on the vectors from the previous section are satisfied in this construction. In particular we note that

$$
\begin{array}{lll}
a_{1}^{T} \eta a_{1}=0, & a_{2}^{T} \eta a_{2}=0, & a_{1}^{T} \eta a_{2}=0, \\
b_{1}^{T} \eta b_{1}=0, & b_{2}^{T} \eta b_{2}=0, & b_{1}^{T} \eta b_{2}=0, \\
a_{1}^{T} b_{1}=0, & a_{2}^{T} b_{2}=0, & a_{1}^{T} b_{2}=-a_{2}^{T} b_{1}=-1+\zeta^{2} .
\end{array}
$$

The above data results in the following matrix,

$$
\mathcal{M}(w)=\left(\begin{array}{cccccccc}
1 & 0 & 0 & 0 & 0 & 0 & 0 & 0 \\
0 & 1 & 0 & 0 & 0 & 0 & 0 & 0 \\
0 & 0 & 1+\frac{2 m(m-w)}{w^{2}-c^{2}} & 0 & 0 & 0 & 0 & \frac{2 a m}{w^{2}-c^{2}} \\
0 & 0 & 0 & 1+\frac{2 m(m-w)}{w^{2}-c^{2}} & 0 & 0 & -\frac{2 a m}{w^{2}-c^{2}} & 0 \\
0 & 0 & 0 & 0 & 1 & 0 & 0 & 0 \\
0 & 0 & 0 & 0 & 0 & 1 & 0 & 0 \\
0 & 0 & 0 & -\frac{2 a m}{w^{2}-c^{2}} & 0 & 0 & 1+\frac{2 m(m+w)}{w^{2}-c^{2}} & 0 \\
0 & 0 & \frac{2 a m}{w^{2}-c^{2}} & 0 & 0 & 0 & 0 & 1+\frac{2 m(m+w)}{w^{2}-c^{2}}
\end{array}\right),
$$

where (at some places) we have replaced $\zeta$ and $c$ in favor of $m$ and $a$. The relations between these parameters are

$$
\zeta=\frac{c-m}{a}, \quad c=\sqrt{m^{2}-a^{2}} .
$$


This matrix is precisely the $\mathrm{SO}(4,4)$ monodromy matrix for the Kerr metric - factorization of it gives the Kerr-field.

Having obtained the monodromy matrix for the Kerr metric, generalization to the four-charge black hole is now straightforward. We simply conjugate the Kerr matrix with the appropriate group element,

$$
\mathcal{M}_{4-\text { charge }}(w)=g^{\#} \mathcal{M}(w) g .
$$

Since in our duality frame, the four-charge black hole corresponds to three-magnetic charges and one-electric charge, we act on $\mathcal{M}(w)$ with the following group element

$$
g=\exp \left[-\delta_{0}\left(E_{q_{0}}+F_{q_{0}}\right)\right] \cdot \exp \left[\delta_{1}\left(E_{p^{1}}+F_{p^{1}}\right)\right] \cdot \exp \left[\delta_{2}\left(E_{p^{2}}+F_{p^{2}}\right)\right] \cdot \exp \left[\delta_{3}\left(E_{p^{3}}+F_{p^{3}}\right)\right] .
$$

The transformed vectors are

$$
\begin{aligned}
& a_{1}=\left(-c_{0} s_{1},-\zeta c_{3} s_{2},-\zeta c_{2} c_{3},-s_{0} s_{1},-c_{1} s_{0},-\zeta c_{2} s_{3}, \zeta s_{2} s_{3}, c_{0} c_{1}\right)^{T} \\
& a_{2}=\left(\zeta c_{0} s_{1}, c_{3} s_{2}, c_{2} c_{3}, \zeta s_{0} s_{1}, \zeta c_{1} s_{0}, c_{2} s_{3},-s_{2} s_{3},-\zeta c_{0} c_{1}\right)^{T} \\
& b_{1}=\left(\zeta c_{0} s_{1},-c_{3} s_{2}, c_{2} c_{3},-\zeta s_{0} s_{1}, \zeta c_{1} s_{0},-c_{2} s_{3},-s_{2} s_{3}, \zeta c_{0} c_{1}\right)^{T} \\
& b_{2}=\left(-c_{0} s_{1}, \zeta c_{3} s_{2},-\zeta c_{2} c_{3}, s_{0} s_{1},-c_{1} s_{0}, \zeta c_{2} s_{3}, \zeta s_{2} s_{3},-c_{0} c_{1}\right)^{T}
\end{aligned}
$$

where to avoid notational clutter we have introduced $c_{i}=\cosh \delta_{i}$ and $s_{i}=\sinh \delta_{i}$. Using these vectors we construct the monodromy matrix of the four-charge black hole. By group property it follows that relations $(4.8 \mathrm{a})-(4.8 \mathrm{c})$ hold as it is. With these choices we find

$$
\begin{aligned}
\gamma_{1} & =\frac{2 \zeta\left(1-\zeta^{2}\right) t_{2}\left(1+t_{1}^{2}\right)}{\left(1+\zeta^{2}\right)\left(t_{1}-t_{2}\right)\left(1+t_{1} t_{2}\right)}, \\
\gamma_{2} & =\frac{2 \zeta\left(1-\zeta^{2}\right) t_{1}\left(1+t_{2}^{2}\right)}{\left(1+\zeta^{2}\right)\left(t_{1}-t_{2}\right)\left(1+t_{1} t_{2}\right)} .
\end{aligned}
$$

From these expressions we readily construct the $\Gamma$ matrix and using relations (3.32a) and (3.32b) we find the $c$ and $d$ vectors, and hence solve the factorization problem. From expressions (3.35) and (3.37) we find the final matrix $M(x)$ for the four-charge black hole.

The conformal factor, which is given by (3.40), takes the form

$$
f^{2}=-4 k_{\mathrm{BM}} t_{1}^{2} t_{2}^{2}\left(1-\zeta^{2}\right)^{2} \frac{\left(1+t_{1} t_{2}\right)^{2}\left(1-\zeta^{2}\right)^{2}-4\left(t_{1}-t_{2}\right)^{2} \zeta^{2}}{\left(1+t_{1}^{2}\right)\left(1+t_{2}^{2}\right)\left(t_{1}-t_{2}\right)^{2}\left(1+t_{1} t_{2}\right)^{2}\left(1+\zeta^{2}\right)^{2} \rho^{2}} .
$$

Using the conformal factor we construct the three-dimensional base metric. Using the base metric and the matrix $M(x)$, we can read off all physical fields. Expressions for these fields are presented in appendix B along with some further details. In this way we recover the full set of fields for the four-charge black hole.

\section{Generalization of BM method: residues of rank $r$}

We now consider the general monodromy matrix

$$
\mathcal{M}(w)=\mathcal{V}^{\#}\left(-\frac{1}{t}, x\right) \mathcal{V}(t, x),
$$


with $\mathcal{V}(t, x)$ the generalization of $V(x) \in \mathrm{G} / \mathrm{K}$ that also depends on the spectral parameter $t$. The map \# $: \mathrm{G} \rightarrow \mathrm{G}$ is the anti-involution already introduced in section 2.1.

For the $N$-soliton solution, one takes $\mathcal{M}(w)$ to be a meromorphic function with $N$ simple poles at $w=w_{k}$ in the form:

$$
\mathcal{M}(w)=\mathbb{1}+\sum_{k=1}^{N} \frac{A_{k}}{w-w_{k}},
$$

and

$$
\mathcal{M}^{-1}(w)=\mathbb{1}-\sum_{k=1}^{N} \frac{B_{k}}{w-w_{k}},
$$

with $A_{k}, B_{k}$ the $x$-independent residue matrices. The $t$-dependent expansions of $\mathcal{M}$ read

$$
\mathcal{M}(t, x)=\mathbb{1}+\sum_{k=1}^{N} \frac{\nu_{k} t_{k} A_{k}}{t-t_{k}}+\sum_{k=1}^{N} \frac{\nu_{k} A_{k}}{1+t t_{k}},
$$

and

$$
\mathcal{M}^{-1}(t, x)=\mathbb{1}-\sum_{k=1}^{N} \frac{\nu_{k} t_{k} B_{k}}{t-t_{k}}-\sum_{k=1}^{N} \frac{\nu_{k} B_{k}}{1+t t_{k}} .
$$

Let $A_{k}, B_{k}$ be diagonalizable matrices of size $n$ and $\operatorname{rank} r,(r \leq n)$, which moreover satisfy $A_{k}=A_{k}^{\#}$ and $B_{k}=B_{k}^{\#}$. There exists a matrix $U_{k}$ satisfying $U_{k}^{-1}=U_{k}^{\#}$ and a diagonal matrix $\Lambda_{k}$ such that

$$
A_{k}=U_{k} \Lambda_{k} U_{k}^{\#}
$$

Thus we can write the matrix $A_{k}$ (same treatment applies to $B_{k}$ ) in the form of a sum of rank one matrices as follows:

$$
A_{k}=\sum_{\alpha=1}^{r} \lambda_{k}^{\alpha} u_{k}^{\alpha} v_{k}^{\alpha T}
$$

where $\lambda_{k}^{\alpha}$ are the non-zero entries of the diagonal matrix $\Lambda_{k}$. The vectors $u_{k}^{\alpha}$ and $v_{k}^{\alpha T}$ are the corresponding ( $n$-dimensional) column vectors of matrix $U_{k}$ and corresponding row vectors of matrix $U_{k}^{\#}$ respectively.

One can write the previous rank one decomposition in a manifestly "\#-invariant" form when the action of the map \# on $g \in \mathrm{G}$ is explicitly known (in the matrix representation of the group). As an example consider the coset space $\mathrm{G} / \mathrm{K}=\mathrm{SO}(4,4) / \mathrm{SO}(2,2) \times \mathrm{SO}(2,2)$ with $\tau$ the involutive automorphism that fixes the subgroup $\mathrm{SO}(2,2) \times \mathrm{SO}(2,2)$. The action of \# on $g \in \mathrm{G}$ is given by $g^{\#}=\eta^{\prime} g^{T} \eta^{\prime}$, with $\eta^{\prime}$ the quadratic form preserved by $\mathrm{SO}(2,2) \times \mathrm{SO}(2,2)$. The residue matrices $A_{k}$ (similarly for $\left.B_{k}\right)$ can be expressed in the form

$$
A_{k}=U_{k} \Lambda_{k} U_{k}^{\#}=U_{k} \eta^{\prime} \Lambda_{k} \eta^{\prime} \eta^{\prime} U^{T} \eta^{\prime}=U_{k} \Lambda_{k}^{\prime} U^{T} \eta^{\prime}=\sum_{\alpha=1}^{r} \lambda_{k}^{\alpha} u_{k}^{\alpha} u_{k}^{\alpha \#}
$$


where we use the "\#-invariance" of the diagonal matrix $\Lambda_{k}$ and $\Lambda_{k}^{\prime}=\eta^{\prime} \Lambda_{k}$. Moreover, the \# operation on column vectors is defined as $u_{k}^{\#}=u_{k}^{T} \eta^{\prime}$ and on row vectors as $u_{k}^{T \#}=\eta^{\prime} u_{k}$. (Indeed, using this definition, we have that for any vector $v$ and a matrix $S=v v^{\#} \in \mathrm{G}$, $S^{\#}=S$ ). Assuming we can adopt this notation in the general case and using the freedom to redefine the vectors and tune $\lambda_{k}^{\alpha}$ accordingly, one can write ${ }^{2}$

$$
A_{k}=\alpha_{k} \sum_{\alpha=1}^{r} p_{k}^{\alpha} p_{k}^{\alpha \#}, \quad B_{k}=\beta_{k} \sum_{\alpha=1}^{r} q_{k}^{\alpha} q_{k}^{\alpha \#}
$$

with $p_{k}^{\alpha}, q_{k}^{\alpha}$ the redefined $n$-dimensional vectors and $\alpha_{k}, \beta_{k}$ are constant parameters, not to be confused with the greek upper indices. The latter enumerate the vectors with respect to the rank of the residue matrix, while the lower indices denoted by $k, l, \ldots$ are the soliton indices and take values in $\{1,2, \ldots, N\}$.

Studying the pole structure of the product $\mathcal{M}(t, x) \mathcal{M}^{-1}(t, x)$ at $t=-\frac{1}{t_{k}}$, one can infer the required conditions on the vectors $p_{k}^{\alpha}, q_{k}^{\alpha}$. The condition for no double poles in the product $\mathcal{M}(t, x) \mathcal{M}^{-1}(t, x)$ at $t=-\frac{1}{t_{k}}$ is fulfilled when

$$
p_{k}^{\alpha \#} q_{k}^{\beta}=0, \quad \text { for all } k \text { and } \alpha=1,2, \ldots, r, \quad \beta=1,2, \ldots, r .
$$

Furthermore, the absence of single poles in $\mathcal{M}(t, x) \mathcal{M}^{-1}(t, x)$ at $t=-\frac{1}{t_{k}}$ requires the condition

$$
\mathcal{A}_{k} B_{k}=A_{k} \mathcal{A}^{k}
$$

to be satisfied, with

$$
\mathcal{A}_{k}=\left.\left(\mathcal{M}(t, x)-\frac{\nu_{k} A_{k}}{1+t t_{k}}\right)\right|_{t \rightarrow-\frac{1}{t_{k}}}, \quad \mathcal{A}^{k}=\left.\left(\mathcal{M}^{-1}(t, x)+\frac{\nu_{k} B_{k}}{1+t t_{k}}\right)\right|_{t \rightarrow-\frac{1}{t_{k}}}
$$

The demand is met if there exist $\gamma_{k}^{\alpha}$ numbers such that

$$
\mathcal{A}_{k} q_{k}^{\alpha}=\nu_{k} \alpha_{k} \gamma_{k}^{\alpha} p_{k}^{\alpha}, \quad p_{k}^{\alpha \#} \mathcal{A}^{k}=\nu_{k} \beta_{k} \gamma_{k}^{\alpha} q_{k}^{\alpha \#}
$$

for all $k=1,2, \ldots, N$ and $\alpha=1,2, \ldots, r$.

The solution of the Riemann-Hilbert problem amounts to the factorization of $\mathcal{M}$, with the expansion (5.4), in the form

$$
\mathcal{M}(w)=A_{-}^{\#}(t, x) M(x) A_{+}(t, x),
$$

\footnotetext{
${ }^{2}$ The notation we have used earlier for the case of $\mathrm{G} / \mathrm{K}=\mathrm{SO}(4,4) / \mathrm{SO}(2,2) \times \mathrm{SO}(2,2)$ is somewhat different. However, the previous notation can be readily translated in the general notation used in this section by identifying $p_{k}^{1}=a_{k}, p_{k}^{2}=-\eta b_{k}, q_{k}^{1}=\eta^{\prime} b_{k}, q_{k}^{2}=\eta \eta^{\prime} a_{k}, \alpha_{k}^{1}=-\beta_{k}^{2}=\alpha_{k}, \alpha_{k}^{2}=-\beta_{k}^{1}=-\beta_{k}, r_{k}^{1}=$ $c_{k}, r_{k}^{2}=\eta d_{k}, s_{k}^{1}=\eta^{\prime} d_{k}, s_{k}^{2}=-\eta \eta^{\prime} c_{k}$ (with $\alpha_{k}, \beta_{k}$ the constants in section 3) and using the \# operation on vectors as defined above.
} 
with $A_{-}(t, x)=A_{+}\left(-\frac{1}{t}, x\right)$ and $M^{\#}(x)=M(x)$. The poles at $t=-\frac{1}{t_{k}}$ come from the factor $A_{+}$and so we assume this matrix to be of the form

$$
A_{+}=\mathbb{1}-\sum_{k=1}^{N} \frac{t C_{k}}{1+t t_{k}}
$$

and

$$
A_{+}^{-1}=\mathbb{1}+\sum_{k=1}^{N} \frac{t D_{k}}{1+t t_{k}}
$$

with $C_{k}=\sum_{\alpha=1}^{r} r_{k}^{\alpha} p_{k}^{\alpha \#}$ and $D_{k}=\sum_{\alpha=1}^{r} q_{k}^{\alpha} s_{k}^{\alpha \#}$. In order to determine the vectors $r_{k}^{\alpha}$, we study the pole structure of the product $A_{+}(t) \mathcal{M}^{-1}(t, x)$ at $t=-\frac{1}{t_{k}}$. The absence of double poles yields the condition

$$
C_{k} B_{k}=0
$$

and is fulfilled when (5.10) holds. The condition for no single poles is

$$
t_{k}^{-2} C_{k} \mathcal{A}^{k}=\left.\left(A_{+}+\frac{t C_{k}}{1+t t_{k}}\right)\right|_{t \rightarrow-\frac{1}{t_{k}}} B_{k} \nu_{k} t_{k}^{-1}
$$

and is satisfied when

$$
q_{k}^{\alpha}=t_{k}^{-1} r_{k}^{\alpha} \gamma_{k}^{\alpha}+\sum_{l \neq k}^{N} \sum_{\beta=1}^{r} \frac{1}{t_{l}-t_{k}} r_{l}^{\beta} p_{l}^{\beta \#} q_{k}^{\alpha}
$$

that is, when these $r N$ vector equations hold. We can express them in a more compact way, in the form ${ }^{3}$

$$
q_{B}=\sum_{A=1}^{r N} r_{A} \Gamma_{A B}
$$

where the capital indices $A, B$ take values in $\{1,2, \ldots, r N\}$ and each value uniquely determines a pair of indices $(k, \alpha)$. This can be done for example through the relations

$$
k=\left\{\begin{array}{lll}
A \bmod N & \text { if } & A \bmod N>0 \\
N & \text { if } & A \bmod N=0,
\end{array} \quad \alpha=1+\left[\frac{A-1}{N}\right],\right.
$$

where [.] denotes the integer part (floor function). The matrix $\Gamma$ is defined as the $r N \times r N$ block matrix with entries

$$
\Gamma_{k l}^{\alpha \beta}=\left\{\begin{array}{lll}
\frac{\gamma_{k}^{\alpha}}{t_{k}} \delta_{\alpha \beta} & \text { for } & k=l \\
\frac{p_{k}^{\alpha} q_{l}^{\beta}}{t_{k}-t_{l}} & \text { for } & k \neq l,
\end{array}\right.
$$

\footnotetext{
${ }^{3}$ These vector equations can be represented by the matrix equation $q=r \Gamma$, where $q$ is the $n \times r N$ matrix whose columns are the vectors $q_{1}^{1}, q_{2}^{1}, \ldots, q_{N}^{1}, q_{1}^{2}, q_{2}^{2}, \ldots, q_{N}^{2}, \ldots, q_{1}^{r}, q_{2}^{r}, \ldots, q_{N}^{r}$ and the matrix $r$ is defined similarly (with columns the $r_{k}^{\alpha}$ vectors).
} 
where the upper indices denote the block entry and the lower indices the entries of each block. It is a symmetric matrix under the condition $p_{k}^{\alpha \#} q_{l}^{\beta}=-p_{l}^{\beta \#} q_{k}^{\alpha}$ for $k \neq l$ and all $\alpha, \beta$ in $\{1,2, \ldots, r\}$. Moreover, when the condition $p_{k}^{\alpha \#} q_{l}^{\beta}=0$ for $k \neq l$ and $\alpha \neq \beta$ holds, the off-diagonal blocks of $\Gamma$ vanish (this is the case in all examples we have worked with so far). Solving (5.20) for the vectors $r_{B}$ we find

$$
r_{B}=\sum_{A=1}^{r N} q_{A}\left(\Gamma^{-1}\right)_{A B} .
$$

There is one more set of vectors that we need to determine and these are the $s_{k}^{\alpha}$ in (5.16). The requirement that $\left(\mathcal{M}(t, x) A_{+}^{-1}\right)^{\#}$ have no poles at $t=-\frac{1}{t_{k}}$ is fulfilled when

$$
p_{k}^{\alpha}=t_{k}^{-1} s_{k}^{\alpha} \gamma_{k}^{\alpha}+\sum_{l \neq k}^{N} \sum_{\beta=1}^{r} \frac{1}{t_{k}-t_{l}} s_{l}^{\beta} p_{k}^{\alpha \#} q_{l}^{\beta} \quad \Longleftrightarrow \quad p_{A}=\sum_{B=1}^{r N} \Gamma_{A B} s_{B}
$$

and the equation for the vectors $s_{A}$ is ${ }^{4}$

$$
s_{A}=\sum_{B=1}^{r N}=\left(\Gamma^{-1}\right)_{A B} p_{B} .
$$

Finally, the matrix $M(x)$ is obtained by

$$
M=A_{+}^{-1}(\infty)=\mathbb{1}+\sum_{A, B=1}^{r N} q_{A} t_{A}^{-1}\left(\Gamma^{-1}\right)_{A B} p_{B}^{\#},
$$

where $t_{A}=t_{k}^{\alpha}=t_{k}$ for all values of $\alpha$.

Conformal factor. The formula for the conformal factor in the multisoliton case with residues of rank $r$ is given by

$$
\begin{aligned}
f^{4} & =k_{\mathrm{BM}} \cdot \operatorname{det} \Gamma \cdot \prod_{A=1}^{r N}\left(t_{A} \nu_{A}\right) \\
& =k_{\mathrm{BM}} \cdot \operatorname{det} \Gamma \cdot \prod_{k=1}^{N}\left(t_{k} \nu_{k}\right)^{r} .
\end{aligned}
$$

This follows by a straightforward application of the computation of appendix A of [13] since the expression for $M$ is formally the same except for the enlarged range for the indices of $\Gamma_{A B}$. The power on $f$ on the left-hand side of (5.27) is due the changed normalization mentioned in footnote 1.

We note that (5.27) is consistent with (3.40) since in the discussion of section 3 the vectors were assumed to satisfy (3.29). In that case the matrix $\Gamma_{A B}$ becomes block diagonal with $r$ repeated blocks of the matrix $\Gamma_{k l}$. Then $\operatorname{det}\left(\Gamma_{A B}\right)=\left(\operatorname{det}\left(\Gamma_{k l}\right)\right)^{r}$ and this leads to the agreement between (3.40) and (5.27) when one takes into account the different powers on $f$.

\footnotetext{
${ }^{4}$ The matrix equation is now $p=s \Gamma^{T}$, where $p, s$ are $n \times r N$ matrices whose columns are the vectors $p_{k}^{\alpha}, s_{k}^{\alpha}$ respectively and are defined similarly to matrices $q$ and $r$.
} 


\section{Discussion}

In this paper we studied the integrability of STU supergravity and proposed an inverse scattering technique for this theory. Our main interest in performing this analysis is to make available solution generating techniques based on integrability for set-ups where the standard BZ construction is not applicable. Our approach makes use of the Geroch group (affine symmetry) of the dimensionally reduced STU theory. We concentrated on Geroch group matrices with simple poles only - the so-called soliton sector. The main difference compared to the $\operatorname{SL}(n, \mathbb{R})$ analysis presented in $[13,14]$ is that in the present $\operatorname{SO}(4,4)$ case the rank of the residue matrices is two - as opposed to one - for simple solutions of physical interest. In view of further generalization (and future applications) of this technique we also presented a generalization to arbitrary group $\mathrm{G}$ incorporating residue matrices of arbitrary rank $r$.

Comparing our solution generating technique to that based on the finite-dimensional G-symmetry used by many authors, we find that it is nicely consistent. A (charging) transformation by a global element $k \in \mathrm{K} \subset \mathrm{G}$ rotates the matrix $\mathcal{M}(w)$ according to (2.19). Since $k$ is $w$-independent it does not affect the location of the poles $w_{k}$ but rotates the residue matrices $A_{k}$ in (5.2) also according to (2.19). This induces a rotation of the vectors arising in the factorization (5.7) but only in such a way that the matrix $\Gamma_{A B}$ does not change and consequently the conformal factor (5.27) is unchanged. The action of the symmetry is then the same that one would have in the three-dimensional system (2.1).

There are many ways in which our study can be extended. The next natural step would be to understand five-dimensional asymptotically flat boundary conditions from the Geroch group point of view. This requires changing the asymptotic behavior of $\mathcal{M}(w)$ for $w \rightarrow \infty$. Together with the results of the present paper, this will allow us to construct the 5d charged rotating Cvetič-Youm [24] metric which in turn will lead to an inverse scattering construction of the JMaRT fuzzball [25]. Such a construction is highly desirable as it will naturally lead to ways to generalize the JMaRT fuzzball. Various problems in relation to five-dimensional black rings will also become accessible once we incorporate five-dimensional asymptotically flat boundary conditions in our formalism. We hope to report on these issues in the near future.

On the technical side there is another difficulty that needs to be overcome before our construction can be applied in its full potential. Recall that, in order to apply our formalism for the construction of the four-charge black hole we used the group property to find the vectors (4.13) starting from that of the Kerr black hole. For this computation, group rotation is sufficient, but we expect that in more complicated situations, in particular for configurations involving three or more poles, one needs to develop some other algorithmic techniques to find appropriate vectors. In this regard, ideas from the interval structure [2628] of gravitational solutions can be useful, but at the moment this remains an open challenging problem.

More generally, since the five-dimensional version of the STU theory has Chern-Simons terms in its Lagrangian, we expect a very large family of non-trivial bubbling — fuzzballlike - solutions [29] to be within reach of our proposed formalism; see [30] for a recent discussion on this point. Although we have taken a significant step forward in attacking 
this problem in this paper, some further technical developments are necessary before such sought after geometries can be explicitly constructed.

\section{Acknowledgements}

We would like to thank Geoffrey Compère for correspondence. AV would also like to thank Albert Einstein Institute, Golm and Institute of Mathematical Sciences, Chennai for their warm hospitality where part of this work was done.

\section{A Conventions}

In this appendix we detail the conventions that we are using for the STU model.

\section{A.1 The $\mathrm{SO}(4,4)$ group and its subgroups}

We adopt the conventions of $[22,31]$. Thus we have the set of $\mathrm{SO}(4,4)$ generators labelled by

$$
H_{\Lambda}, \quad E_{\Lambda}, \quad F_{\Lambda}, \quad E_{q_{\Lambda}}, \quad F_{q_{\Lambda}}, \quad E_{p^{\Lambda}}, \quad F_{p^{\Lambda}}
$$

for $\Lambda=0,1,2,3$. The subgroup relevant to time-like reductions is $\mathrm{SO}(2,2) \times \mathrm{SO}(2,2) \approx$ $\mathrm{SL}(2)^{4}$; it is generated by

$$
K_{\Lambda}=E_{\Lambda}-F_{\Lambda}, \quad K_{q_{\Lambda}}=E_{q_{\Lambda}}+F_{q_{\Lambda}}, \quad K_{p^{\Lambda}}=E_{p^{\Lambda}}+F_{p^{\Lambda}} .
$$

The four commuting sets of $\mathrm{SL}(2)$ generators in standard basis are for example given by

$$
\begin{aligned}
h_{0} & =\frac{1}{2}\left(-K_{q_{0}}+K_{p^{1}}+K_{p^{2}}+K_{p^{3}}\right), \\
h_{1} & =\frac{1}{2}\left(+K_{q_{0}}-K_{p^{1}}+K_{p^{2}}+K_{p^{3}}\right), \\
h_{2} & =\frac{1}{2}\left(+K_{q_{0}}+K_{p^{1}}-K_{p^{2}}+K_{p^{3}}\right), \\
h_{3} & =\frac{1}{2}\left(+K_{q_{0}}+K_{p^{1}}+K_{p^{2}}-K_{p^{3}}\right), \\
e_{0} & =\frac{1}{4}\left(-K_{0}+K_{1}+K_{2}+K_{3}+K_{q_{1}}+K_{q_{2}}+K_{q_{3}}+K_{p^{0}}\right), \\
f_{0} & =\frac{1}{4}\left(+K_{0}-K_{1}-K_{2}-K_{3}+K_{q_{1}}+K_{q_{2}}+K_{q_{3}}+K_{p^{0}}\right), \\
e_{1} & =\frac{1}{4}\left(+K_{0}-K_{1}+K_{2}+K_{3}+K_{q_{1}}-K_{q_{2}}-K_{q_{3}}+K_{p^{0}}\right), \\
f_{1} & =\frac{1}{4}\left(-K_{0}-K_{1}-K_{2}-K_{3}+K_{q_{1}}-K_{q_{2}}-K_{q_{3}}+K_{p^{0}}\right), \\
e_{2} & =\frac{1}{4}\left(+K_{0}-+K_{1}-K_{2}+K_{3}-K_{q_{1}}+K_{q_{2}}-K_{q_{3}}+K_{p^{0}}\right), \\
f_{2} & =\frac{1}{4}\left(-K_{0}-K_{1}+K_{2}-K_{3}-K_{q_{1}}+K_{q_{2}}-K_{q_{3}}+K_{p^{0}}\right), \\
e_{3} & =\frac{1}{4}\left(+K_{0}+K_{1}+K_{2}-K_{3}-K_{q_{1}}-K_{q_{2}}-K_{q_{3}}+K_{p^{0}}\right), \\
f_{3} & =\frac{1}{4}\left(-K_{0}-K_{1}-K_{2}+K_{3}-K_{q_{1}}-K_{q_{2}}+K_{q_{3}}+K_{p^{0}}\right) .
\end{aligned}
$$


We write the $\mathrm{SO}(4,4)$ group element in Borel gauge as ${ }^{5}$

$$
V=e^{-U H_{0}} \cdot\left[\prod_{I=1,2,3}\left(e^{-\frac{1}{2} \log y^{I} H_{I}} e^{-x^{I} E_{I}}\right)\right] \cdot e^{-\zeta^{\Lambda} E_{q_{\Lambda}}-\tilde{\zeta}_{\Lambda} E_{p^{\Lambda}}} \cdot e^{-\sigma E_{0}} .
$$

Next, we will explain how the scalar fields appearing in this coset element are related to the physical quantities of the STU model.

\section{A.2 Four-dimensional metric and duality relations in $D=3$}

We parameterise the four-dimensional metric as

$$
d s_{4}^{2}=-e^{2 U}\left(d t+\omega_{3}\right)^{2}+e^{-2 U} d s_{3}^{2} .
$$

The three-dimensional metric $d s_{3}^{2}$ in turn is given by (2.7).

The $D=3$ vector fields obtained by reduction from $D=4$ are defined by

$$
A^{\Lambda}=\zeta^{\Lambda}\left(d t+\omega_{3}\right)+A_{3}^{\Lambda},
$$

which also defines the scalars $\zeta^{\Lambda}$. As for any reduction of an $\mathcal{N}=2$ supergravity theory, the duality relations between vector and scalar fields in $D=3$ are

$$
d \sigma-\frac{1}{2}\left(\zeta^{\Lambda} d \tilde{\zeta}_{\Lambda}-\tilde{\zeta}_{\Lambda} d \zeta^{\Lambda}\right)=-e^{4 U} \star d \omega_{3}
$$

and

$$
-d \tilde{\zeta}_{\Lambda}=e^{2 U}(\operatorname{Im} N)_{\Lambda \Sigma} \star\left(d A_{3}^{\Sigma}+\zeta^{\Sigma} d \omega_{3}\right)+(\operatorname{Re} N)_{\Lambda \Sigma} d \zeta^{\Sigma}
$$

The matrix $N_{\Lambda \Sigma}$ is defined through the cubic prepotential $F(X)=-\frac{X^{1} X^{2} X^{3}}{X^{0}}$ via

$$
N_{\Lambda \Sigma}=\bar{F}_{\Lambda \Sigma}+2 i \frac{(\operatorname{Im} F)_{\Lambda \Xi}(\operatorname{Im} F)_{\Sigma \Pi} X^{\Xi} X^{\Pi}}{(\operatorname{Im} F)_{\Xi \Pi} X^{\Xi} X^{\Pi}}
$$

where subscripts $F_{\Lambda}$ denote derivatives of $F$ with respect to $X^{\Lambda}$. In the gauge $X^{0}=1$ the scalar fields are (for $I=1,2,3$ )

$$
z^{I}=\frac{X^{I}}{X^{0}}=X^{I}=x^{I}+i y^{I} .
$$

In the present case these definitions imply (we lower the indices on $x^{I}$ for readability)

$$
(\operatorname{Re} N)_{\Lambda \Sigma}=\left(\begin{array}{cccc}
-2 x_{1} x_{2} x_{3} & x_{2} x_{3} & x_{1} x_{3} & x_{1} x_{2} \\
x_{2} x_{3} & 0 & -x_{3} & -x_{2} \\
x_{1} x_{3} & -x_{3} & 0 & -x_{1} \\
x_{1} x_{2} & -x_{2} & -x_{1} & 0
\end{array}\right)
$$

\footnotetext{
${ }^{5}$ Note that the normalisation of $\sigma$ is changed compared to [31].
} 
and

$$
(\operatorname{Im} N)_{\Lambda \Sigma}=\left(\begin{array}{cccc}
\frac{-x_{3}^{2} y_{1}^{2} y_{2}^{2}-x_{1}^{2} y_{3}^{2} y_{2}^{2}-x_{2}^{2} y_{1}^{2} y_{3}^{2}-y_{1}^{2} y_{2}^{2} y_{3}^{2}}{y_{1} y_{2} 2_{3}} & \frac{x_{1} y_{2} y_{3}}{y_{1}} & \frac{x_{2} y_{1} y_{3}}{y_{2}} & \frac{x_{3} y_{1} y_{2}}{y_{3}} \\
\frac{x_{1} y_{2} y_{3}}{y_{1}} & -\frac{y_{2} y_{3}}{y_{1}} & 0 & 0 \\
\frac{x_{2} y_{1} y_{3}}{y_{2}} & 0 & -\frac{y_{1} y_{3}}{y_{2}} & 0 \\
\frac{x_{3} y_{2} y_{2}}{y_{3}} & 0 & 0 & -\frac{y_{1} y_{2}}{y_{3}}
\end{array}\right),
$$

with inverse

$$
\left((\operatorname{Im} N)^{-1}\right)_{\Lambda \Sigma}=\frac{1}{y_{1} y_{2} y_{3}}\left(\begin{array}{cccc}
-1 & -x_{1} & -x_{2} & -x_{3} \\
-x_{1} & -x_{1}^{2}-y_{1}^{2} & -x_{1} x_{2} & -x_{1} x_{3} \\
-x_{2} & -x_{1} x_{2} & -x_{2}^{2}-y_{2}^{2} & -x_{2} x_{3} \\
-x_{3} & -x_{1} x_{3} & -x_{2} x_{3} & -x_{3}^{2}-y_{3}^{2}
\end{array}\right) .
$$

\section{B Two-dimensional fields for the four-charge black hole}

In this appendix we show how to obtain the four-charge solution of Cvetič-Youm from $\mathcal{V}(t)$ and $V$ that were constructed in section 4 .

The first thing to do is to change coordinates on the two-dimensional base. This is done by parameterizing the pole values of the spectral parameter through

$$
\begin{aligned}
& t_{1}=\frac{(u-c)(1+v)}{\sqrt{\left(u^{2}-c^{2}\right)\left(1-v^{2}\right)}}, \\
& t_{2}=\frac{(u+c)(1+v)}{\sqrt{\left(u^{2}-c^{2}\right)\left(1-v^{2}\right)}} .
\end{aligned}
$$

As a next step we change from the prolate spherical coordinates $(u, v)$ to the BoyerLindquist coordinates $(r, x)$ defined by

$$
u=r-m, \quad v=x .
$$

The constants $\zeta$ and $c$ that appear in the parameterisations of the pole and residue vectors are conveniently given in terms of $m$ and $a$ as

$$
\zeta=\frac{c-m}{a}, \quad c=\sqrt{m^{2}-a^{2}} .
$$

Now we introduce the abbreviations

$$
\Delta=\frac{r^{2}+a^{2} x^{2}-2 m r}{r^{2}+a^{2} x^{2}}, \quad \sigma_{\text {Kerr }}=-\frac{2 m a x}{r^{2}+a^{2} x^{2}} .
$$

We again stress the factor of 2 for $\sigma$ for Kerr compared to [31]. Using the conformal factor (4.15), the three-dimensional base metric is here found to be

$$
d s_{3}^{2}=\frac{r^{2}-2 m r+a^{2} x^{2}}{r^{2}-2 m r+a^{2}} d r^{2}+\left(r^{2}-2 m r+a^{2} x^{2}\right) \frac{d x^{2}}{1-x^{2}}+\left(1-x^{2}\right)\left(r^{2}-2 m r+a^{2}\right) d \varphi^{2} .
$$


We have fixed the normalization factor in (4.15) to be $k_{\mathrm{BM}}=-4 c^{2} \frac{\left(1+\zeta^{2}\right)^{2}}{\left(1-\zeta^{2}\right)^{4}}=-\frac{m^{2} a^{4}}{c^{2}(m-c)^{2}}$ by the requirement of asymptotic flatness.

The presentation of the rest of the fields below is closely related to that of [17]. The scalar fields $x_{I}$ of (A.10) are given by

$$
\begin{aligned}
& x_{1}=\frac{\left(c_{01} s_{23}-s_{01} c_{23}\right) \sigma_{\text {Kerr }}}{h_{2} h_{3}+s_{23}^{2} \sigma_{\text {Kerr }}^{2}}, \\
& x_{2}=\frac{\left(c_{02} s_{13}-s_{02} c_{13}\right) \sigma_{\text {Kerr }}}{h_{1} h_{3}+s_{13}^{2} \sigma_{\text {Kerr }}^{2}}, \\
& x_{3}=\frac{\left(c_{03} s_{12}-s_{03} c_{12}\right) \sigma_{\text {Kerr }}}{h_{1} h_{2}+s_{12}^{2} \sigma_{\text {Kerr }}^{2}} .
\end{aligned}
$$

Introducing in addition the shorthand

$$
\begin{aligned}
h_{i} & =\left(c_{i}^{2}-s_{i}^{2} \Delta\right) \\
c_{i_{1} \ldots i_{n}} & =\cosh \delta_{i_{1}} \ldots \cosh \delta_{i_{n}} \\
s_{i_{1} \ldots i_{n}} & =\sinh \delta_{i_{1}} \ldots \sinh \delta_{i_{n}}
\end{aligned}
$$

the scalar fields $y_{I}$ of (A.10) are found to be

$$
\begin{aligned}
& y_{1}=\frac{W}{h_{2} h_{3}+s_{23}^{2} \sigma_{\mathrm{Kerr}}^{2}} \\
& y_{2}=\frac{W}{h_{1} h_{3}+s_{13}^{2} \sigma_{\mathrm{Kerr}}^{2}} \\
& y_{3}=\frac{W}{h_{1} h_{2}+s_{12}^{2} \sigma_{\mathrm{Kerr}}^{2}},
\end{aligned}
$$

where

$$
\begin{gathered}
W^{2}=h_{0} h_{1} h_{2} h_{3}+\sigma_{\text {Kerr }}^{2}\left(2 c_{0123} s_{0123}-\left(s_{012}^{2}+s_{013}^{2}+s_{023}^{2}+s_{123}^{2}+4 s_{0123}^{2}\right) \Delta\right. \\
\left.+2 s_{0123}^{2} \Delta^{2}\right)+s_{0123}^{2} \sigma_{\text {Kerr }}^{4}
\end{gathered}
$$

In terms of (B.9) and (B.4) the dilaton of the $D=4$ to $D=3$ reduction is given by

$$
e^{2 U}=\frac{\Delta}{W}
$$

The dual of the Kaluza-Klein vector of the reduction reads

$$
\sigma=\frac{\sigma_{\text {Kerr }}}{2 W^{2}}\left\{c_{0123}\left[2+(1-\Delta)\left(\sum_{i=0}^{3} s_{i}^{2}\right)\right]+s_{0123}\left[\left(2+\sum_{i=0}^{3} s_{i}^{2}\right)\left(\Delta^{2}-\Delta+\sigma_{\text {Kerr }}^{2}\right)-2 \Delta\right]\right\} .
$$

The scalars coming from the vector multiplets are

$$
\begin{aligned}
& \tilde{\zeta}_{0}=\frac{\sigma_{\text {Kerr }}}{W^{2}}\left[h_{0}\left(s_{0} c_{123}-c_{0} s_{123} \Delta\right)+s_{0} c_{0} s_{0123} \sigma_{\text {Kerr }}^{2}\right], \\
& \zeta^{1}=\frac{\sigma_{\text {Kerr }}}{W^{2}}\left[h_{1}\left(s_{1} c_{023}-c_{1} s_{023} \Delta\right)+s_{1} c_{1} s_{0123} \sigma_{\text {Kerr }}^{2}\right],
\end{aligned}
$$




$$
\begin{aligned}
& \zeta^{2}=\frac{\sigma_{\text {Kerr }}}{W^{2}}\left[h_{2}\left(s_{2} c_{013}-c_{2} s_{013} \Delta\right)+s_{2} c_{2} s_{0123} \sigma_{\text {Kerr }}^{2}\right], \\
& \zeta^{3}=\frac{\sigma_{\text {Kerr }}}{W^{2}}\left[h_{3}\left(s_{3} c_{012}-c_{3} s_{012} \Delta\right)+s_{3} c_{3} s_{0123} \sigma_{\text {Kerr }}^{2}\right],
\end{aligned}
$$

and

$$
\begin{aligned}
& \zeta^{0}=+\left\{\frac{c_{0}}{s_{0}}-\frac{1}{s_{0} W^{2}}\left(c_{0} h_{1} h_{2} h_{3}+\left(s_{0} c_{123}-c_{0} s_{123} \Delta\right) s_{123} \sigma_{\text {Kerr }}^{2}\right)\right\} \\
& \tilde{\zeta}_{1}=-\left\{\frac{c_{1}}{s_{1}}-\frac{1}{s_{1} W^{2}}\left(c_{1} h_{0} h_{2} h_{3}+\left(s_{1} c_{023}-c_{1} s_{023} \Delta\right) s_{023} \sigma_{\text {Kerr }}^{2}\right)\right\} \\
& \tilde{\zeta}_{2}=-\left\{\frac{c_{2}}{s_{2}}-\frac{1}{s_{2} W^{2}}\left(c_{2} h_{0} h_{1} h_{3}+\left(s_{2} c_{013}-c_{2} s_{013} \Delta\right) s_{013} \sigma_{\text {Kerr }}^{2}\right)\right\} \\
& \tilde{\zeta}_{3}=-\left\{\frac{c_{3}}{s_{3}}-\frac{1}{s_{3} W^{2}}\left(c_{3} h_{0} h_{1} h_{2}+\left(s_{3} c_{012}-c_{3} s_{012} \Delta\right) s_{012} \sigma_{\text {Kerr }}^{2}\right)\right\} .
\end{aligned}
$$

Upon substituting the expressions for $\sigma_{\text {Kerr }}$ and $\Delta$ and after performing the dualizations using (A.7) and (A.8), the above expressions take the following form

$$
\begin{aligned}
& x_{1}=2 \max \frac{s_{01} c_{23}-c_{01} s_{23}}{r_{2} r_{3}+a^{2} x^{2}}, \\
& x_{2}=2 \max \frac{s_{02} c_{13}-c_{02} s_{13}}{r_{1} r_{3}+a^{2} x^{2}}, \\
& x_{3}=2 \max \frac{s_{03} c_{12}-c_{03} s_{12}}{r_{1} r_{2}+a^{2} x^{2}},
\end{aligned}
$$

where $r_{i}=r+2 m s_{i}^{2}$, and

$$
\begin{aligned}
y_{1} & =\frac{\tilde{W}}{r_{2} r_{3}+a^{2} x^{2}}, \\
y_{2} & =\frac{\tilde{W}}{r_{1} r_{3}+a^{2} x^{2}}, \\
y_{3} & =\frac{\tilde{W}}{r_{1} r_{2}+a^{2} x^{2}} .
\end{aligned}
$$

with $\tilde{W}^{2}:=\left(r^{2}+a^{2} x^{2}\right)^{2} W^{2}$ given below in (B.23). The scalars appearing in (A.6) are

$$
\begin{aligned}
\zeta^{0} & =\frac{2 m c_{0} s_{0}\left(r_{1} r_{2} r_{3}+r a^{2} x^{2}\right)+4 a^{2} m^{2} x^{2} e_{0}}{\tilde{W}^{2}}, \\
\zeta^{1} & =-2 \max \frac{\left(s_{1} c_{023}-c_{1} s_{023}\right)\left(r r_{1}+a^{2} x^{2}\right)+2 m c_{1} s_{023} r_{1}}{\tilde{W}^{2}}, \\
\zeta^{2} & =-2 \max \frac{\left(s_{2} c_{013}-c_{2} s_{013}\right)\left(r r_{2}+a^{2} x^{2}\right)+2 m c_{2} s_{013} r_{2}}{\tilde{W}^{2}}, \\
\zeta^{3} & =-2 \max \frac{\left(s_{3} c_{012}-c_{3} s_{012}\right)\left(r r_{3}+a^{2} x^{2}\right)+2 m c_{3} s_{012} r_{3}}{\tilde{W}^{2}},
\end{aligned}
$$

where

$$
e_{0}=\left(c_{0}^{2}+s_{0}^{2}\right) c_{123} s_{123}-c_{0} s_{0}\left(s_{12}^{2}+s_{23}^{2}+s_{13}^{2}+2 s_{123}^{2}\right) \text {. }
$$


The three dimensional one-forms read with (A.7) and (A.8)

$$
\omega_{3}=2 a m\left(1-x^{2}\right) \frac{\left(c_{0123} r-(r-2 m) s_{0123}\right)}{r^{2}-2 m r+a^{2} x^{2}} d \varphi
$$

and

$$
\begin{aligned}
& A_{3}^{0}=-2 a m\left(1-x^{2}\right) \frac{\left(s_{0} c_{123} r-(r-2 m) c_{0} s_{123}\right)}{r^{2}-2 m r+a^{2} x^{2}} d \varphi \\
& A_{3}^{1}=2 m s_{1} c_{1} x \frac{r^{2}+a^{2}-2 m r}{r^{2}-2 m r+a^{2} x^{2}} d \varphi \\
& A_{3}^{2}=2 m s_{2} c_{2} x \frac{r^{2}+a^{2}-2 m r}{r^{2}-2 m r+a^{2} x^{2}} d \varphi \\
& A_{3}^{3}=2 m s_{3} c_{3} x \frac{r^{2}+a^{2}-2 m r}{r^{2}-2 m r+a^{2} x^{2}} d \varphi .
\end{aligned}
$$

Finally,

$$
\begin{aligned}
\tilde{W}^{2}=r_{0} r_{1} r_{2} r_{3}+a^{4} x^{4}+a^{2} x^{2}[ & 2 r^{2}+2 m r\left(s_{0}^{2}+s_{1}^{2}+s_{2}^{2}+s_{3}^{2}\right) \\
+ & \left.8 m^{2} c_{0123} s_{0123}-4 m^{2}\left(s_{012}^{2}+s_{123}^{2}+s_{023}^{2} s_{013}^{2}+2 s_{0123}^{2}\right)\right] .
\end{aligned}
$$

Using these expressions the four-dimensional metric and the various matter fields can be readily obtained by substitution into (A.5) and (A.6). In these expressions $a$ is the bare rotation parameter and $m$ is the bare mass parameter.

Open Access. This article is distributed under the terms of the Creative Commons Attribution License (CC-BY 4.0), which permits any use, distribution and reproduction in any medium, provided the original author(s) and source are credited.

\section{References}

[1] V.A. Belinsky and V.E. Zakharov, Integration of the Einstein Equations by the Inverse Scattering Problem Technique and the Calculation of the Exact Soliton Solutions, Sov. Phys. JETP 48 (1978) 985 [Zh. Eksp. Teor. Fiz. 75 (1978) 1953] [INSPIRE].

[2] V.A. Belinsky and V.E. Sakharov, Stationary Gravitational Solitons with Axial Symmetry, Sov. Phys. JETP 50 (1979) 1 [Zh. Eksp. Teor. Fiz. 77 (1979) 3] [INSPIRE].

[3] V. Belinski and E. Verdaguer, Gravitational solitons, Cambridge Univ. Press, U.K., 2001.

[4] R. Emparan and H.S. Reall, Black Holes in Higher Dimensions, Living Rev. Rel. 11 (2008) 6 [arXiv:0801.3471] [INSPIRE].

[5] H. Iguchi, K. Izumi and T. Mishima, Systematic solution-generation of five-dimensional black holes, Prog. Theor. Phys. Suppl. 189 (2011) 93 [arXiv:1106.0387] [INSPIRE].

[6] J.V. Rocha, M.J. Rodriguez, O. Varela and A. Virmani, Charged black rings from inverse scattering, Gen. Rel. Grav. 45 (2013) 2099 [arXiv:1305.4969] [INSPIRE].

[7] J. Ehlers, Transformations of static exterior solutions of Einstein's gravitational field equations into different solutions by means of conformal mappings, in Les Théories Physiques de la Gravitation, CNRS, Paris, 1959, pg. 275. 
[8] E. Cremmer and B. Julia, The SO(8) Supergravity, Nucl. Phys. B 159 (1979) 141 [inSPIRE].

[9] N. Marcus and J.H. Schwarz, Three-Dimensional Supergravity Theories, Nucl. Phys. B 228 (1983) 145 [INSPIRE].

[10] P. Breitenlohner, D. Maison and G.W. Gibbons, Four-Dimensional Black Holes from Kaluza-Klein Theories, Commun. Math. Phys. 120 (1988) 295 [INSPIRE].

[11] P. Figueras, E. Jamsin, J.V. Rocha and A. Virmani, Integrability of Five Dimensional Minimal Supergravity and Charged Rotating Black Holes, Class. Quant. Grav. 27 (2010) 135011 [arXiv: 0912.3199] [INSPIRE].

[12] P. Breitenlohner and D. Maison, On the Geroch Group, Annales Poincaré Phys. Theor. 46 (1987) 215 [INSPIRE].

[13] D. Katsimpouri, A. Kleinschmidt and A. Virmani, Inverse Scattering and the Geroch Group, JHEP 02 (2013) 011 [arXiv: 1211.3044] [INSPIRE].

[14] P. Breitenlohner and D. Maison, Solitons in Kaluza-Klein Theories, unpublished notes, June 1986.

[15] M.J. Duff, J.T. Liu and J. Rahmfeld, Four-dimensional string-string-string triality, Nucl. Phys. B 459 (1996) 125 [hep-th/9508094] [INSPIRE].

[16] K. Behrndt, R. Kallosh, J. Rahmfeld, M. Shmakova and W.K. Wong, STU black holes and string triality, Phys. Rev. D 54 (1996) 6293 [hep-th/9608059] [INSPIRE].

[17] Z.-W. Chong, M. Cvetič, H. Lü and C.N. Pope, Charged rotating black holes in four-dimensional gauged and ungauged supergravities, Nucl. Phys. B 717 (2005) 246 [hep-th/0411045] [INSPIRE].

[18] M. Cvetič, G.W. Gibbons, C.N. Pope and Z.H. Saleem, Electrodynamics of Black Holes in STU Supergravity, arXiv:1310.5717 [INSPIRE].

[19] D.D.K. Chow and G. Compère, Seed for general rotating non-extremal black holes of $N=8$ supergravity, Class. Quant. Grav. 31 (2014) 022001 [arXiv:1310.1925] [INSPIRE].

[20] M. Cvetič and D. Youm, Entropy of nonextreme charged rotating black holes in string theory, Phys. Rev. D 54 (1996) 2612 [hep-th/9603147] [INSPIRE].

[21] G. Bossard, H. Nicolai and K.S. Stelle, Universal BPS structure of stationary supergravity solutions, JHEP 07 (2009) 003 [arXiv: 0902.4438] [INSPIRE].

[22] G. Bossard, Y. Michel and B. Pioline, Extremal black holes, nilpotent orbits and the true fake superpotential, JHEP 01 (2010) 038 [arXiv: 0908.1742] [INSPIRE].

[23] H. Nicolai, Two-dimensional gravities and supergravities as integrable system, Lect. Notes Phys. 396 (1991) 231, in Proceedings of Recent aspects of quantum fields, pg. 43, Schladming, May 1991.

[24] M. Cvetič and D. Youm, General rotating five-dimensional black holes of toroidally compactified heterotic string, Nucl. Phys. B 476 (1996) 118 [hep-th/9603100] [INSPIRE].

[25] V. Jejjala, O. Madden, S.F. Ross and G. Titchener, Non-supersymmetric smooth geometries and D1 - D5 - P bound states, Phys. Rev. D 71 (2005) 124030 [hep-th/0504181] [INSPIRE].

[26] T. Harmark, Stationary and axisymmetric solutions of higher-dimensional general relativity, Phys. Rev. D 70 (2004) 124002 [hep-th/0408141] [INSPIRE]. 
[27] S. Hollands and S. Yazadjiev, Uniqueness theorem for 5-dimensional black holes with two axial Killing fields, Commun. Math. Phys. 283 (2008) 749 [arXiv:0707.2775] [InSPIRE].

[28] Y. Chen and E. Teo, Rod-structure classification of gravitational instantons with $\mathrm{U}(1) \times \mathrm{U}(1)$ isometry, Nucl. Phys. B 838 (2010) 207 [arXiv: 1004.2750] [INSPIRE].

[29] S.D. Mathur, The Fuzzball proposal for black holes: An Elementary review, Fortsch. Phys. 53 (2005) 793 [hep-th/0502050] [INSPIRE].

[30] I. Bena and N.P. Warner, Resolving the Structure of Black Holes: Philosophizing with a Hammer, arXiv:1311.4538 [INSPIRE].

[31] A. Virmani, Subtracted Geometry From Harrison Transformations, JHEP 07 (2012) 086 [arXiv: 1203.5088] [INSPIRE]. 